\title{
Low Temperature Ferromagnetism in Chemically Ordered FeRh Nanocrystals
}

\author{
A. Hillion, ${ }^{1}$ A. Cavallin, ${ }^{2}$ S. Vlaic, ${ }^{2}$ A. Tamion, ${ }^{1}$ F. Tournus, ${ }^{1}$ G. Khadra, ${ }^{1}$ J. Dreiser,${ }^{3}$ C. Piamonteze,${ }^{3}$ F. Nolting, ${ }^{3}$ \\ S. Rusponi, ${ }^{2}$ K. Sato, ${ }^{4}$ T. J. Konno, ${ }^{4}$ O. Proux,${ }^{5}$ V. Dupuis, ${ }^{1}$ and H. Brune ${ }^{2}$ \\ ${ }^{1}$ Laboratoire de Physique de la Matière Condensée et Nanostructures, UMR-CNRS 5586 and Université Lyon 1, \\ 69622 Villeurbanne cedex, France \\ ${ }^{2}$ Institute of Condensed Matter Physics, Ecole Polytechnique Fédérale de Lausanne (EPFL), 1015 Lausanne, Switzerland \\ ${ }^{3}$ Swiss Light Source, Paul Scherrer Institut, 5232 Villigen, Switzerland \\ ${ }^{4}$ Institute for Materials Research, Tohoku University, 2-1-1 Katahira, Sendai 980-8577, Japan \\ ${ }^{5}$ Observatoire des Sciences de l'Univers, 38051 Grenoble, France
}

(Received 7 November 2012; published 21 February 2013)

\begin{abstract}
In sharp contrast to previous studies on FeRh bulk, thin films, and nanoparticles, we report the persistence of ferromagnetic order down to $3 \mathrm{~K}$ for size-selected $3.3 \mathrm{~nm}$ diameter nanocrystals embedded into an amorphous carbon matrix. The annealed nanoparticles have a $B 2$ structure with alternating atomic Fe and Rh layers. X-ray magnetic dichroism and superconducting quantum interference device measurements demonstrate ferromagnetic alignment of the $\mathrm{Fe}$ and $\mathrm{Rh}$ magnetic moments of 3 and $1 \mu_{\mathrm{B}}$, respectively. The ferromagnetic order is ascribed to the finite-size induced structural relaxation observed in extended $\mathrm{x}$-ray absorption spectroscopy.
\end{abstract}

DOI: 10.1103/PhysRevLett.110.087207

PACS numbers: 75.30.Kz, 75.75.Cd, 81.07.Bc

Iron-rhodium alloys exhibit competing ferromagnetic (FM) and antiferromagnetic (AFM) phases with transition temperatures close to ambient for nearly equiatomic composition and body-centered-cubic (bcc) $\mathrm{CsCl}$-like $B 2$ structure. The competition between the two magnetic orders of FeRh holds great potential in spintronics and heat assisted magnetic recording [1,2]. Moreover, the peculiar bulk FeRh magnetic phase diagram enables its use as active material in heat pumps and refrigerators [3-5].

At ambient conditions, bulk $B 2 \mathrm{FeRh}$ is a $G$-type AFM with a total magnetic moment on the iron atoms of $3.3 \mu_{\mathrm{B}}$ and no appreciable moment on the rhodium atoms [6-8]. Above the transition temperature of $370 \mathrm{~K}$, the atomic moments of $\mathrm{Fe}$ and $\mathrm{Rh}$ are ferromagnetically aligned and take on total values of 3.2 and $0.9 \mu_{\mathrm{B}}$, respectively [6-8]. While it has long been known that the bcc unit cell volume expands by $\approx 1 \%$ upon transforming to FM order [9], recent experiments suggest that distortions of the bcc structure may occur [10]. Given the itinerant character of the $3 d$ electrons, the coupling between crystallographic and magnetic order in this system is both rich and very delicate as demonstrated by the theoretical challenge to model the system [11], as well as by recent pump-probe experiments focusing on ultrafast magnetization control [12].

Finite-size systems of this alloy have received particular attention by their potential to stabilize the FM phase at room temperature and below. Strained thin films [13,14] showed traces of a FM phase down to $300 \mathrm{~K}$, while $a b$ initio calculations predicted FM down to $0 \mathrm{~K}$ for a Rh-terminated 9 ML FeRh(001) film [15] and for 8-atom $\mathrm{FeRh}$ clusters [16]. Indeed, since nanosized crystals may present significantly different interatomic distances and unit cell distortions with respect to bulk $[17,18]$, a fundamentally modified magnetic phase diagram can be expected for FeRh nanocrystals. However, the first experiments on chemically synthesized FeRh nanoparticles (NPs) failed to evidence low temperature stability of the FM phase. Most notably, they raised important questions, such as partial $B 2$ ordering, elemental segregation, and coalescence upon annealing [19-21].

In this Letter, we demonstrate the persistence of FM order down to below $3 \mathrm{~K}$ in size-selected FeRh nanocrystals with a mean diameter of $3.3 \mathrm{~nm}$ that are embedded into a carbon $(\mathrm{C})$ matrix and thus protected from pollution and coalescence. Both structural and magnetic properties dramatically change upon annealing of the NPs. While the as-deposited ones are in a chemically disordered fcc structure, the annealed NPs are in the chemically ordered $B 2$ phase with alternating atomic $\mathrm{Fe}$ and $\mathrm{Rh}$ planes, as evidenced by high resolution transmission electron microscopy (HRTEM). X-ray magnetic circular dichroism (XMCD) reveals for particles in the $B 2$ phase FM order between $\mathrm{Fe}$ and $\mathrm{Rh}$ with magnetic moments of 3 and $1 \mu_{\mathrm{B}}$, respectively. Combined $\mathrm{XMCD}$ and superconducting quantum interference device (SQUID) magnetometry demonstrate that our 1400-atom NPs are single magnetic domain with a magnetic volume identical to the geometric one and a blocking temperature of around $12 \mathrm{~K}$. The $\mathrm{x}$-ray absorption spectroscopy (XAS) line shape at the Fe $L_{3}$ edge consists of a single peak, thus excluding chemical interactions with the C-matrix atoms as a possible source of the FM order. Based on extended X-ray absorption fine structure (EXAFS) analysis at the Fe $K$ edge, we ascribe the observed FM order to finite-size induced structural relaxation in which the mean interatomic distances are 
the bulk values, but their distribution widths are larger by more than $20 \%$ with respect to the bulk distance distribution.

FeRh NPs are synthesized as follows: a plasma created by the impact of a laser (YAG, $\lambda=532 \mathrm{~nm}$, pulse duration $=8 \mathrm{~ns}$ ) on a FeRh target is thermalized by injection of a continuous flow of helium at low pressure (30 mbar) inducing the cluster growth [22]. Clusters are subsequently cooled down in the supersonic expansion taking place at the exit nozzle of the source, mass-selected by an electrostatic quadrupole, and transferred to an ultrahigh vacuum chamber (base pressure of $5 \times 10^{-10}$ Torr) where they are deposited at low kinetic energy together with carbon atoms onto a carbon buffer. TEM grids have been prepared with $7 \%$ surface concentration, whereas magnetically characterized samples had $1 \%$ vol. to suppress coalescence and magnetic interactions. The asdeposited particles have an fcc structure and have been transformed into the $B 2$ structure by annealing at $970 \mathrm{~K}$ for $1 \mathrm{~h}$ at a background pressure of $p<10^{-6}$ mbar. The HRTEM images after annealing have been acquired operating a FEI Titan 80-300 TEM at $300 \mathrm{kV}$ with a field emission gun and an aberration corrector for the objective lens $[23,24]$ whereas images before annealing have been acquired on a Jeol 2010F at $200 \mathrm{kV}$. XAS and XMCD measurements were performed in the total electron yield mode with a magnetic field parallel to the $\mathrm{x}$-ray beam and perpendicular to the sample surface at the X-Treme beam line of the Swiss Light Source [25]. EXAFS experiments at the Fe $K$ edge $(7112 \mathrm{eV})$ were performed at room temperature at the CRG-BM30b-FAME beam line at ESRF [26]. SQUID measurements were performed using a commercial instrument (Quantum Design, MPMS-XL5). The chemical composition of the NPs has been investigated by means of energy $\mathrm{x}$-ray dispersive spectroscopy revealing a composition of $\mathrm{Fe}_{x} \mathrm{Rh}_{1-x}$ with $x=0.51$.

Figure 1 shows HRTEM images, XAS, and XMCD measurements of the B2-FeRh nanocrystals. The remarkable size homogeneity is documented in Fig. 1(a), while Figs. 1(b) and 1(c) show the crystalline order of particles imaged along the [001] and [110] directions, respectively. The average NP diameter is $3.3 \mathrm{~nm}$, and the size distribution has a relative dispersion of $10 \%$. The Fourier transforms present well defined [100] superlattice spots indicative of alternating $\mathrm{Rh}$ and Fe planes. Ball models of the biatomic $B 2$ rhombic dodecahedra are shown in the respective orientation in the right column. The NPs are perfectly crystalline with a $B 2$ structure and a lattice constant of $a=3.0 \pm 0.2 \AA$; within the error bar this is in agreement with bulk $B 2 \mathrm{FeRh}$ [9]. To our knowledge, this is the first time that chemical order is observed in equiatomic FeRh NPs of that size.

The XAS and resulting XMCD spectra reported in Figs. 1(d) and 1(e) exhibit strong dichroism for both atomic species and reveal that their moments are
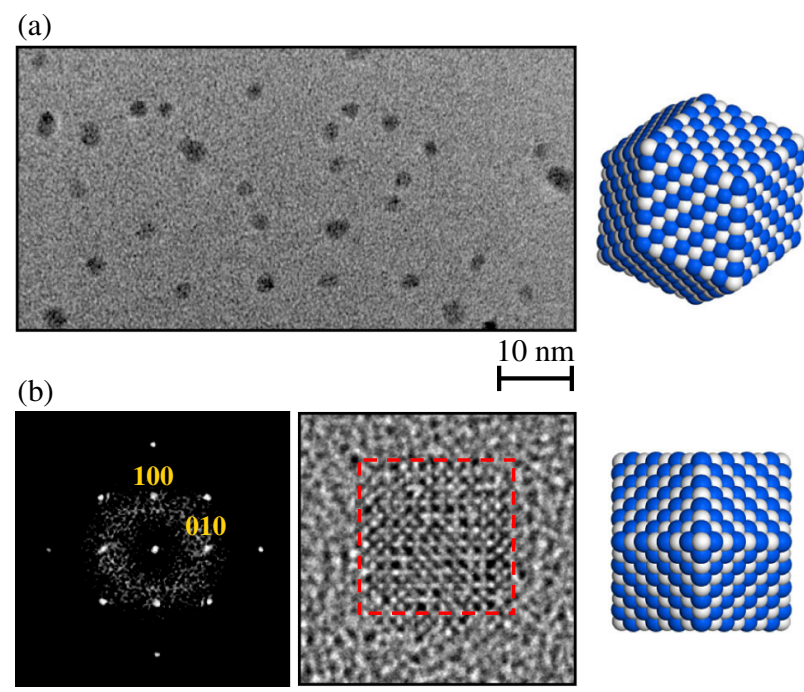

(c)
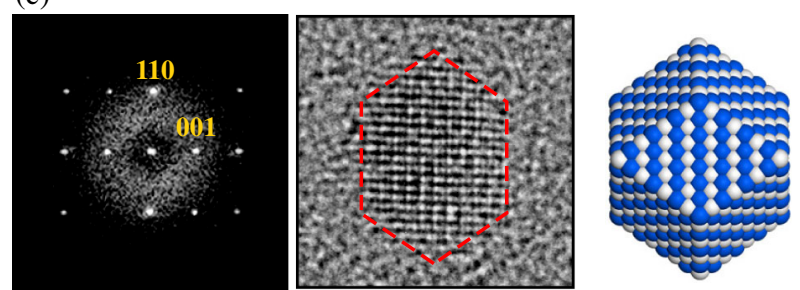

$3.2 \mathrm{~nm}$

(d)

(e)
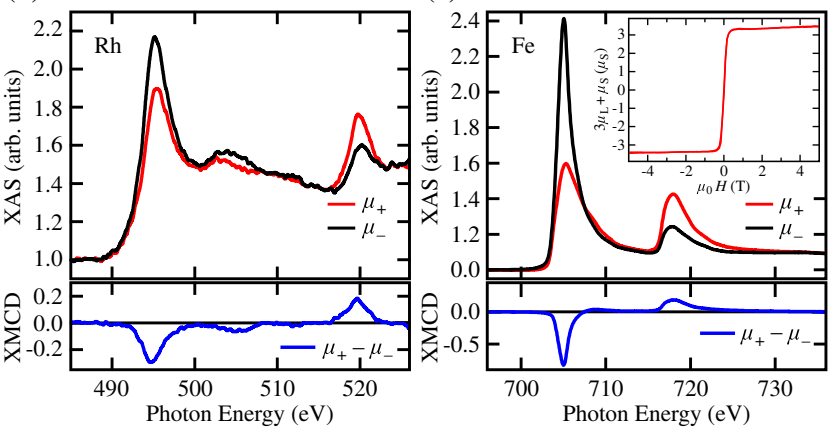

FIG. 1 (color online). (a) Large scale TEM image of $B 2$-FeRh NPs embedded in a carbon matrix. (b) and (c) HRTEM images of two such NPs viewed along the [001] and [110] directions, their Fourier transforms, and ball models. (d) and (e) XAS and XMCD spectra at the Rh $M_{2,3}$ and $\mathrm{Fe} L_{2,3}$ edges acquired at $T=3 \mathrm{~K}$ with a magnetic field $\mu_{0} H=5 \mathrm{~T}$ along the beam direction. Inset in (e) $M(H)$ measured at the Fe $L_{3}$ edge at $T=3 \mathrm{~K}$.

ferromagnetically aligned because their XMCD has the same sign. The orbital and spin magnetic moments per $\mathrm{Rh}$ and $\mathrm{Fe}$ atom are given in Table I and have been derived using the sum rules [27,28] with bulk number of $d$ holes, $h_{d}=3.51$ for Fe and $h_{d}=2.34$ for Rh [29]. The magnetic moments are comparable with those observed in bulk FeRh above the transition temperature. The observation of bulklike mean magnetic moments excludes the possibility of AFM particles having uncompensated surface spins, as then these atoms would have unrealistically large magnetic 
TABLE I. Atomic orbital $\mu_{L}$ and spin $\mu_{S}$ magnetic moments at $T=3 \mathrm{~K}$ and $\mu_{0} H=5 \mathrm{~T}$ for $B 2-F e R h$ NPs.

\begin{tabular}{lccc}
\hline \hline & $\mu_{L}\left(\mu_{\mathrm{B}}\right)$ & $\mu_{S}\left(\mu_{\mathrm{B}}\right)$ & $\mu_{L}+\mu_{S}\left(\mu_{\mathrm{B}}\right)$ \\
\hline $\mathrm{Rh}$ & $0.18 \pm 0.03$ & $0.8 \pm 0.2$ & $1.0 \pm 0.2$ \\
$\mathrm{Fe}$ & $0.25 \pm 0.03$ & $2.7 \pm 0.3$ & $3.0 \pm 0.3$ \\
\hline \hline
\end{tabular}

moments (note that in the considered NPs, approximately $35 \%$ of the atoms are at the surface). Because the samples are made of randomly oriented nanocrystals, the magnetic dipole term $\mu_{T}$, reflecting the aspherical spin moment distribution around the absorbing atom, averages to zero. Therefore, the effective spin moment evaluated from the $\mathrm{XMCD}$ signal is the true magnetic spin moment. Notice that the Rh absorption spectra present in addition to the $M_{2}$ and $M_{3}$ edges a feature at $503 \mathrm{eV}$ that has been reported before $[29,30]$. In contrast to these references, we observe a small dichroic signal also for this peak. Because it is unclear how this signal enters into the sum rules, its area has been considered as contributing to the uncertainty on the estimated Rh moments.

Figure 2 shows the results for the as-deposited NPs. The HRTEM images reveal their fcc structure, the two examples shown in Figs. 2(a) and 2(b) are representative for the more than 50 investigated particles. The mean diameter of $3.4 \pm 0.2 \mathrm{~nm}$ is within the error identical to that of $B 2$ NPs. The lattice constant is $a=3.7 \pm 0.2 \AA$. The XMCD measurements reveal significantly smaller magnetic moments of $1.3 \mu_{\mathrm{B}}$ and $0.2 \mu_{\mathrm{B}}$ per $\mathrm{Fe}$ and $\mathrm{Rh}$ atom; see Figs. 2(c) and 2(d) and Table II, reminiscent of inhomogeneous or noncollinear magnetic order in the nanoparticles. Note the small shoulder of the Fe absorption line at $+1.6 \mathrm{eV}$ with respect to the main $L_{3}$ edge. It is absent for the annealed particles and might be due to the different chemical environment of $\mathrm{Fe}$ atoms at the NP-C interface, or to interstitial $\mathrm{C}$ atoms [31]. Its disappearance upon annealing would accordingly be attributed to graphitization of the amorphous $\mathrm{C}$ matrix at the NP surface [32,33], or to segregation of $\mathrm{C}$ interstitials due to the increased mixing enthalpy in the bcc phase [34]. In fact, the enthalpy of formation of an interstitial impurity is close to zero in $\mathrm{Fe}$ fcc but it is larger than $1 \mathrm{eV}$ in $\mathrm{Fe}$ bcc and increases reducing the particle size [34,35], thus leading to $\mathrm{C}$-free NPs upon annealing. These results make also clear why a $\mathrm{C}$ matrix represents a good choice for this study. The magnetism of clean FeRh nanocrystals can only be explored if the embedding matrix prevents coalescence, contamination, and strong hybridization of the NP surface with the matrix atoms. As alternative matrix materials, one could imagine metal oxides. A criterion for negligible oxidation at the NPs surface is an oxygen affinity of the metallic component of the oxide larger than that of Fe [36]. With this criterion in mind we see that for two largely used oxides, i.e., $\mathrm{Al}_{2} \mathrm{O}_{3}$ and $\mathrm{MgO}$, the former would be a better candidate.
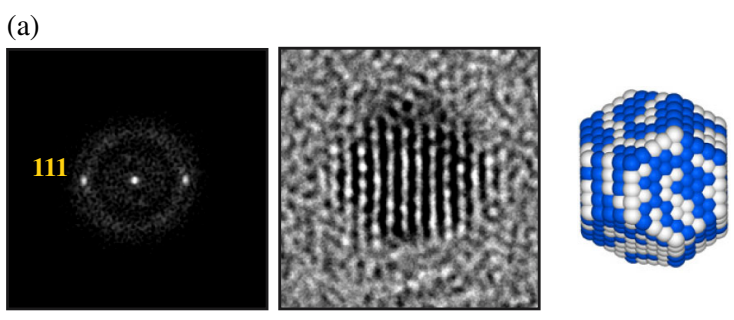

(b)
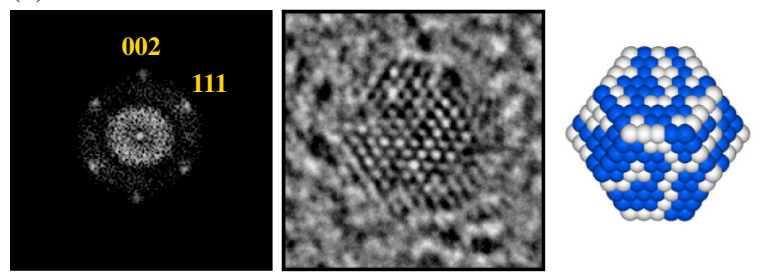

(c)

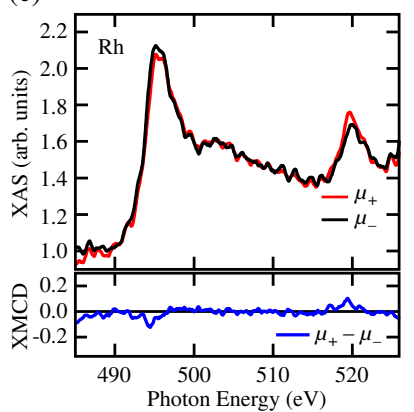

(d)

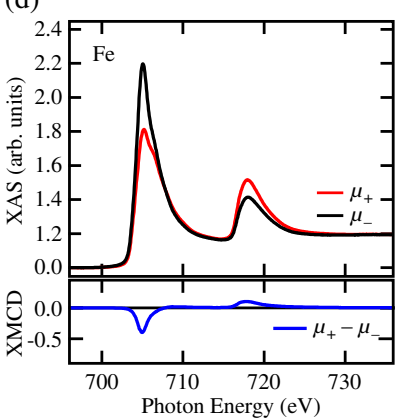

FIG. 2 (color online). (a) and (b) HRTEM images of two asdeposited NPs presented together with their Fourier transforms and ball models with random atomic arrangement. (c) and (d) XAS and XMCD measurements at the Rh $M_{2,3}$ and Fe $L_{2,3}$ edges, respectively, acquired at $T=3 \mathrm{~K}$ while applying a magnetic field $\mu_{0} H=5 \mathrm{~T}$ along the beam direction.

Additional information on the magnetic properties of the NPs is gained from the SQUID measurements shown in Fig. 3. The zero-field-cooling (ZFC) and field-cooling (FC) magnetization curves show a crossover from superparamagnetism to blocking characteristic of FM NPs. The magnetic size and effective anisotropy have been determined by the simultaneous fit of ZFC, FC, and $M(H)$ at $100 \mathrm{~K}$ [37]. We used the semianalytical model presented in Ref. [38] and the magnetic moments of Tables I and II as input. For both phases, the mean magnetic diameter coincides within the error with the geometric one; see Table III. This confirms negligible particle coalescence and testifies the unquenched magnetism at

TABLE II. Atomic orbital and spin magnetic moments at $T=$ $3 \mathrm{~K}$ and $\mu_{0} H=5 \mathrm{~T}$ for as-deposited FeRh NPs.

\begin{tabular}{lccc}
\hline \hline & $\mu_{L}\left(\mu_{\mathrm{B}}\right)$ & $\mu_{S}\left(\mu_{\mathrm{B}}\right)$ & $\mu_{L}+\mu_{S}\left(\mu_{\mathrm{B}}\right)$ \\
\hline $\mathrm{Rh}$ & $0.00 \pm 0.05$ & $0.2 \pm 0.2$ & $0.2 \pm 0.2$ \\
$\mathrm{Fe}$ & $0.08 \pm 0.02$ & $1.2 \pm 0.1$ & $1.3 \pm 0.1$ \\
\hline \hline
\end{tabular}



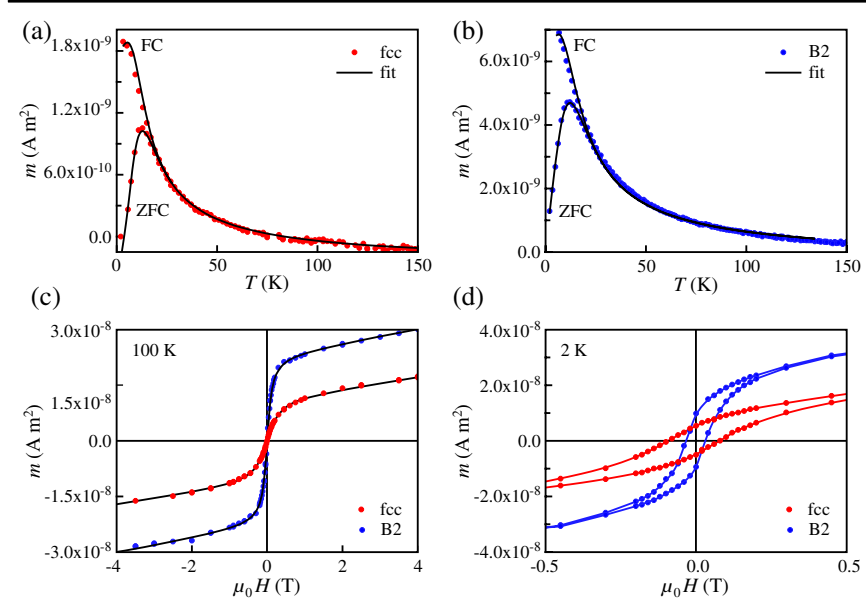

(d)

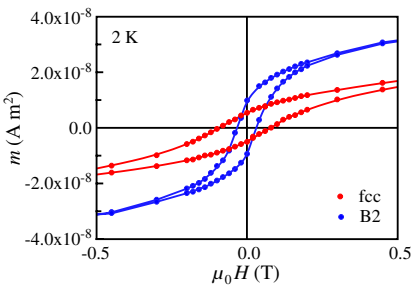

FIG. 3 (color online). (a) and (b) SQUID ZFC and FC magnetization $M(T)$ of fcc and $B 2$ samples, respectively. Solid lines show best fits with the parameters listed in Table III. (c) and (d) magnetization curves $M(H)$ at $100 \mathrm{~K}$ and $2 \mathrm{~K}$, respectively.

the NPs surface. Both $B 2$ and fcc ZFC curves peak around $T_{m}=12 \mathrm{~K}$, and the effective magnetic anisotropies $K$ of both phases are around $130 \mathrm{~kJ} / \mathrm{m}^{3}$. The magnetization curves acquired at $100 \mathrm{~K}$ and at $2 \mathrm{~K}$ are presented in Figs. 3(c) and 3(d). Above the blocking temperature, we observe the typical superparamagnetic fully reversible behavior without a coercive field, while at $T=2 \mathrm{~K}$, the coercive field measures 80 and $35 \mathrm{mT}$ for fcc and $B 2 \mathrm{NPs}$, respectively. The effect of chemical ordering is immediately visible: the saturation magnetization $M_{s}$ is two times larger for the $B 2$ NPs in good agreement with XMCD experiments, while the coercive field decreases by the same amount. Not surprisingly, the $H_{c} M_{s}$ product is nearly conserved through the fcc- $B 2$ transformation, since it is proportional to the almost constant $K$.

We further tested the coupling between crystallographic structure and magnetic order by EXAFS measurements at the Fe $K$ edge. Figure 4 presents the data together with best fits from multiple-scattering path expansion [39] using the Artemis software [40]. Data were simulated after a 1.2-3.2 A Fourier-window filtering (distances uncorrected from phase shift). The fits then return the number of nearest neighbors to the absorbing $\mathrm{Fe}$ atom for each element $N$, their average distances $R$, and their Debye-Waller factors $\sigma^{2}$, which are related to the bond-length dispersion; see

TABLE III. Temperature where the ZFC magnetization takes on its maximum $T_{m}$, mean magnetic diameter $D_{m}$, relative dispersion $w$, and effective anisotropy energy $K$ determined by a fit of $\mathrm{ZFC}, \mathrm{FC}$, and $M(H)$ at $100 \mathrm{~K}$, as well as coercive fields $\left(\mu_{0} H_{c}\right)$ determined from $M(H)$ at $2 \mathrm{~K}$.

\begin{tabular}{lccccc}
\hline \hline & $T_{m}(K)$ & $D_{m}(\mathrm{~nm})$ & $w(\%)$ & $K\left(\mathrm{~kJ} / \mathrm{m}^{3}\right)$ & $\mu_{0} H_{c}(\mathrm{mT})$ \\
\hline fcc & 12 & $3.3 \pm 0.2$ & $15 \pm 3$ & $127 \pm 15$ & 80 \\
$B 2$ & 12 & $3.3 \pm 0.2$ & $15 \pm 3$ & $133 \pm 15$ & 35 \\
\hline \hline
\end{tabular}

(a)

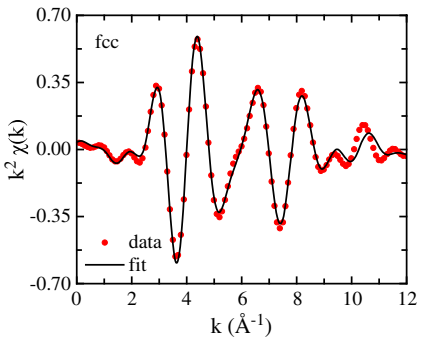

(b)

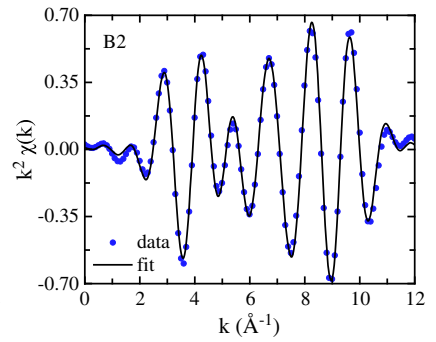

FIG. 4 (color online). (a) and (b) Fe $K$-edge EXAFS data at $300 \mathrm{~K}$ (dots, Fourier-window-filtered to select contributions from 1.2 to $3.2 \AA$ ) for the fcc and $B 2$ samples together with fits (lines) using the parameters in Table IV.

Table IV. As-deposited NPs present $N_{\mathrm{Rh}} / N_{\mathrm{Fe}} \approx 1$, further confirming the chemically disordered fcc structure with an average unit cell volume of $(3.64 \AA)^{3}$. Annealing the NPs has profound implications on the shape of EXAFS signal; see Fig. 4. The spectra can in this case be modeled with a first-neighbor ratio $N_{\mathrm{Rh}} / N_{\mathrm{Fe}}$ equal to $4 / 3$ and a distance ratio $R_{\mathrm{Rh}} / R_{\mathrm{Fe}}$ equal to $\sqrt{3} / 2$, as expected for the $B 2$ phase. The unit cell volume $(2.98 \AA)^{3}$ coincides with that of bulk $B 2 \mathrm{FeRh}$ [8]. The number of $\mathrm{C}$ atoms neighboring the emitting $\mathrm{Fe}$ is reduced upon annealing, in agreement with XMCD measurements. Debye-Waller factors are more than $20 \%$ larger than in bulk [10], possibly due to the lattice parameter relaxation close to the NP surface. Because competition between AFM and FM is very sensitive to the crystallographic structure in FeRh, such finitesize induced structural relaxation might be at the origin of the stable FM order observed down to $T=3 \mathrm{~K}$.

Our combined structural and magnetic study demonstrates that annealed $3.3 \mathrm{~nm}$ FeRh NPs are in the $B 2$ phase presenting alternating $\mathrm{Fe}$ and $\mathrm{Rh}$ atomic planes. Furthermore, these NPs are FM at $T=3 \mathrm{~K}$ and thus do not present the AFM-FM transition characteristic of bulk and thin film specimens. Atomic orbital and spin magnetic moments are compatible with those observed in bulk above the transition temperature and indicate perfect FM alignment. This first observation of low temperature

TABLE IV. Parameters obtained from multiple-scattering path-expansion fits of the EXAFS Fe $K$-edge data. With respect to the emitting Fe atoms one obtains element specific nearestneighbor numbers $N$, their distances $R$, and their Debye-Waller factors $\sigma^{2}$. Top shows as-deposited fcc and bottom annealed $B 2$ NPs.

\begin{tabular}{ccccc}
\hline \hline & & $N$ & $R(\AA)$ & $\sigma^{2}\left(\AA^{2}\right)$ \\
\hline fcc & $\mathrm{Fe}$ & $4.3 \pm 0.4$ & $2.56 \pm 0.01$ & $0.020 \pm 0.001$ \\
& $\mathrm{Rh}$ & $4.3 \pm 0.4$ & $2.59 \pm 0.01$ & $0.010 \pm 0.001$ \\
& $\mathrm{C}$ & $1.6 \pm 0.2$ & $1.98 \pm 0.01$ & $0.006 \pm 0.001$ \\
$B 2$ & $\mathrm{Fe}$ & $4.4 \pm 0.4$ & $2.98 \pm 0.01$ & $0.010 \pm 0.001$ \\
& $\mathrm{Rh}$ & $5.8 \pm 0.6$ & $2.57 \pm 0.01$ & $0.007 \pm 0.001$ \\
& $\mathrm{C}$ & $0.7 \pm 0.1$ & $2.00 \pm 0.01$ & $0.006 \pm 0.001$ \\
\hline \hline
\end{tabular}


stabilization of the FM phase in nanoscale FeRh crystals suggests a rich size-dependent magnetic phase diagram and paves the road for the creation of larger FeRh NPs switching from AFM to FM at tunable temperatures, pressures, and magnetic fields.

The authors acknowledge C. Albin, O. Boisron, F. Donati, Q. Dubout, and L. Gragnaniello for their technical support and for fruitful discussions, PLYRA, CML, and the personnel of the X-Treme and FAME beam lines for their technical support, as well as funding from COST "nanoalloys" MP0903, from the Swiss National Science Foundation, and from the Sino-Swiss Science and Technology Cooperation Project No. IZLCZ2 123892.

[1] J.-U. Thiele, S. Maat, and E.E. Fullerton, Appl. Phys. Lett. 82, 2859 (2003).

[2] Z. Jia, N. Seetala, and R. Misra, Physica (Amsterdam) 405, 2189 (2010).

[3] M. P. Annaorazov, M. Ünal, S. A. Nikitin, A. L. Tyurin, and K. A. Asatryan, J. Magn. Magn. Mater. 251, 61 (2002).

[4] M. E. Gruner and P. Entel, Phase Transit. 78, 209 (2005).

[5] M. Manekar and S. B. Roy, J. Phys. D 41, 192004 (2008).

[6] M. Fallot, Ann. Phys. (N.Y.) 10, 291 (1938).

[7] E. Bertaut, A. Delapalme, F. Forrat, G. Roult, F. de Bergevin, and R. Pauthenet, J. Appl. Phys. 33, 1123 (1962).

[8] G. Shirane, C. W. Chen, P. A. Flinn, and R. Nathans, Phys. Rev. 131, 183 (1963).

[9] F. de Bergevin and L. Muldawer, C.R. Hebd. Seances Acad. Sci. 252, 1347 (1961).

[10] T. Miyanaga, T. Itoga, T. Okazaki, and K. Nitta, J. Phys. Conf. Ser. 190, 012097 (2009).

[11] P. M. Derlet, Phys. Rev. B 85, 174431 (2012).

[12] S. O. Mariager, F. Pressacco, G. Ingold, A. Caviezel, E. Möhr-Vorobeva, P. Beaud, S. L. Johnson, C. J. Milne, E. Mancini, S. Moyerman, E. E. Fullerton, R. Feidenhans'1, C. H. Back, and C. Quitmann, Phys. Rev. Lett. 108, 087201 (2012).

[13] I. Suzuki, T. Koike, M. Itoh, T. Taniyama, and T. Sato, J. Appl. Phys. 105, 07E501 (2009).

[14] R. Fan, C. J. Kinane, T. R. Charlton, R. Dorner, M. Ali, M. A. de Vries, R. M. D. Brydson, C. H. Marrows, B.J. Hickey, D. A. Arena, B. K. Tanner, G. Nisbet, and S. Langridge, Phys. Rev. B 82, 184418 (2010).

[15] S. Lounis, M. Benakki, and C. Demangeat, Phys. Rev. B 67, 094432 (2003).

[16] J. H. Mokkath and G. M. Pastor, Phys. Rev. B 85, 054407 (2012).

[17] F. Baletto and R. Ferrando, Rev. Mod. Phys. 77, 371 (2005).

[18] R. Ferrando, J. Jellinek, and R. L. Johnston, Chem. Rev. 108, 845 (2008).

[19] H. Y. Y. Ko and T. Suzuki, J. Appl. Phys. 101, 09J103 (2007).
[20] D. Ciuculescu, C. Amiens, M. Respaud, A. Falqui, P. Lecante, R.E. Benfield, L. Jiang, K. Fauth, and B. Chaudret, Chem. Mater. 19, 4624 (2007).

[21] Z. Jia, J. W. Harrell, and R. D. K. Misra, Appl. Phys. Lett. 93, 022504 (2008).

[22] A. Perez, P. Melinon, V. Dupuis, L. Bardotti, B. Masenelli, F. Tournus, B. Prevel, J. Tuaillon-Combes, E. Bernstein, A. Tamion, N. Blanc, D. Tainoff, O. Boisron, G. Guiraud, M. Broyer, M. Pellarin, N. D. Fatti, F. Vallee, E. Cottancin, J. Lerme, J.-L. Vialle, C. Bonnet, P. Maioli, A. Crut, C. Clavier, J.L. Rousset, and F. Morfin, Int. J. Nanotechnology 7, 523 (2010).

[23] K. W. Urban, Science 321, 506 (2008).

[24] C. J. Hetherington, L.-Y. S. Chang, S. Haigh, P. D. Nellist, L. C. Gontard, R. E. Dunin-Borkowski, and A. I. Kirkland, Microsc. Microanal. 14, 60 (2008).

[25] C. Piamonteze, U. Flechsig, S. Rusponi, J. Dreiser, J. Heidler, M. Schmidt, R. Wetter, M. Calvi, T. Schmidt, H. Pruchova, J. Krempasky, C. Quitmann, H. Brune, and F. Nolting, J. Synchrotron Radiat. 19, 661 (2012).

[26] O. Proux, V. Nassif, A. Prat, O. Ulrich, E. Lahera, X. Biquard, J. J. Menthonnex, and J. L. Hazemann, J. Synchrotron Radiat. 13, 59 (2006).

[27] B. T. Thole, P. Carra, F. Sette, and G. van der Laan, Phys. Rev. Lett. 68, 1943 (1992).

[28] P. Carra, B. T. Thole, M. Altarelli, and X. Wang, Phys. Rev. Lett. 70, 694 (1993).

[29] C. Stamm, J.-U. Thiele, T. Kachel, I. Radu, P. Ramm, M. Kosuth, J. Minár, H. Ebert, H. A. Dürr, W. Eberhardt, and C. H. Back, Phys. Rev. B 77, 184401 (2008).

[30] V. Sessi, K. Kuhnke, J. Zhang, J. Honolka, K. Kern, C. Tieg, O. Šipr, J. Minár, and H. Ebert, Phys. Rev. B 82, 184413 (2010).

[31] C. Kuivila, J. Butt, and P. Stair, Appl. Surf. Sci. 32, 99 (1988).

[32] N. Jaouen, D. Babonneau, J. M. Tonnerre, D. Carbone, F. Wilhelm, A. Rogalev, T. K. Johal, and G. van der Laan, Phys. Rev. B 76, 104421 (2007).

[33] A. Tamion, M. Hillenkamp, A. Hillion, F. Tournus, J. Tuaillon-Combes, O. Boisron, S. Zafeiratos, and V. Dupuis, J. Appl. Phys. 110, 063904 (2011).

[34] A. Tolvanen, A. V. Krasheninnikov, A. Kuronen, and K. Nordlund, Phys. Status Solidi (c) 7, 1274 (2010).

[35] A. R. Harutyunyan, N. Awasthi, A. Jiang, W. Setyawan, E. Mora, T. Tokune, K. Bolton, and S. Curtarolo, Phys. Rev. Lett. 100, 195502 (2008).

[36] A. Lehnert, S. Rusponi, M. Etzkorn, S. Ouazi, P. Thakur, and H. Brune, Phys. Rev. B 81, 104430 (2010).

[37] A. Tamion, M. Hillenkamp, F. Tournus, E. Bonet, and V. Dupuis, Appl. Phys. Lett. 95, 062503 (2009).

[38] F. Tournus and A. Tamion, J. Magn. Magn. Mater. 323, 1118 (2011).

[39] S. I. Zabinsky, J. J. Rehr, A. Ankudinov, R. C. Albers, and M. J. Eller, Phys. Rev. B 52, 2995 (1995).

[40] B. Ravel and M. Newville, J. Synchrotron Radiat. 12, 537 (2005). 